\title{
Study on Toll Plaza Design Based on M/M/1 Queue Theory
}

\section{Cheng Wang}

\author{
School of Mathematics and Economics, Hubei University of Education, Wuhan 430205, P. R. \\ China \\ wangc80@163.com
}

\begin{abstract}
Keywords: Toll plaza design; M/M/1 queue theory; NSGA-II
\end{abstract}
\begin{abstract}
In this paper, an improved model base on $\mathrm{M} / \mathrm{M} / 1$ queue theory is presented to design the toll plaza by considering different ways of charging in the actual toll service system (human-staffed, automated and electronic toll collection), and the Non-dominated Sorting Genetic Algorithm II (NSGA-II) is used to optimize the design of lane number and charging mode layout. Then the numbers of the three tollbooths are determined and the corresponding optimal designing scheme of the fan-out area before the barrier toll is obtained.
\end{abstract}

\section{Introduction}

The design of tollbooths has become a global concern. Moreover, there are a great number of reports and research from various countries, where the definition of capacities and service levels, and the shape of tollbooths are the crux problems. Among many international studies, most of them applied queuing theory and analogue simulation. For example, Abdelwahab [1] used traffic microsimulation model to optimize the long queues during high traffic hour, and used VB.NET to simulate the whole process from drivers entering the tool station. Usha et al. [2] applied crowd sourcing to maintain waiting time for each tollbooth to obtain the best tollbooth in a toll plaza. Both of them considered the waiting time of the queues, by which they proposed a time saving method.

Based on the theoretical analysis method, Cheng [3] analyzed the actual traffic situation and presented a set of optimization design of ETC and MTC hybrid toll station. Zhao [4] collected field data to analyze and evaluate the impact of the ETC capacity and the number of toll channel openings on toll station capacity, which proved the superiority of ETC channel in traffic capacity. Liu et al. [5] established a queuing theory model for highway toll station design which combined with the characteristics of highway toll collection system, thus a reasonable solution to solve the toll station design is provided. Based on the analysis of the characteristics of freeway toll station traffic flow, Liao [6] established a toll station queuing model and calculated the toll capacity of different number of ETC lanes under MTC vehicle conditions and multi-lane conditions. Through the study of the simulation research of toll station comprehensive service ability, Li et al. [7] came to the toll station service ability when the ETC traffic occupies under the condition of different proportion in the case of a certain amount of traffic input. Feng et al. [8] proposed a kind of comprehensive evaluation system of highway toll station layout based on the theory of the fuzzy evaluation, which provided a scientific and reasonable basis for the selection of toll station layout system. Corwin et al. [9] simulated realistic conditions. For high traffic density, the optimal number of tollbooths exceeds the number of highway lanes by about $50 \%$, while for low traffic density the optimal number of tollbooths equals the number of lanes.

In this paper, we will present an improved model base on $\mathrm{M} / \mathrm{M} / 1$ queue theory to design the toll plaza by considering different ways of charging in the actual toll service system (human-staffed, automated and electronic toll collection), and use the Non-dominated Sorting Genetic Algorithm II (NSGA-II) to optimize the design of lane number and charging mode layout.

\section{The Model}

In order to study the capacities for tollbooths, here we must use a mathematical method to describe the specific characteristics of traffic flow. In reality, the arrival of vehicles is random. 
According to the principle of traffic flow, the traffic flow on highways is smaller than that on urban roads. Thus, the arrival distribution of vehicles can be regarded as a Poisson distribution. And based on the existing investigation and study, the charging time complies with a Normal distribution. In the queuing system of a barrier tool, as vehicles are in a queue for each channel, we will establish a single-channel queuing model M/M/1 [14] and evaluate the capacity of each toll channel by computing the spending time of each vehicle in the toll system. At last, we can select an appropriate toll channel combination based these results of the single-channel queuing model.

Let $\lambda$ be the average number of vehicles arriving in unit time, and $\mu$ be the average number of vehicles served in unit time. Then the service intensity of queuing system of toll station can be expressed as

$$
\rho=\frac{\lambda}{\mu}
$$

The distribution law of queue length $X$ can be expressed as

$$
\pi_{k}=(1-\rho) \rho^{k}, k=0,1,2, \cdots \text {. }
$$

The distribution law of waiting queue length can be expressed as

$$
\left\{\begin{array}{l}
P\left\{X_{q}=0\right\}=\pi_{0}+\pi_{1}=1-\rho^{2} \\
P\left\{X_{q}=0\right\}=\pi_{k+1}=(1-\rho) \rho^{k+1}, k=1,2,3, \cdots
\end{array}\right.
$$

and the average value of waiting queue length is

$$
E\left(X_{q}\right)=\frac{\rho^{2}}{1-\rho}
$$

and the expected waiting time is

$$
E(W)=\frac{\rho}{\mu(1-\rho)}=\frac{E\left(X_{q}\right)}{\lambda}
$$

The expected service time can be expressed as

$$
E(B)=\frac{1}{\mu}
$$

The expectedresidence time, which means the expected waiting time plus the expected service time, i.e.,

$$
\begin{aligned}
& E(T)=E(W)+E(B)=\frac{E(X)}{\lambda}, \\
& E(T)=\frac{1}{\lambda} \cdot \frac{\rho^{2}}{1-\rho}+\frac{1}{\mu}=\frac{1}{\mu-\lambda} .
\end{aligned}
$$

Since the utilization rate and traffic capacity of three types of toll channels are different, that is, the number of vehicles arriving in unit time and the number of vehicles served in unit time are not the same, so we respectively consider the queuing system for each barrier tool, and obtain the average waiting time for each tollbooth. Then the average time that vehicles wait in the whole barrier tool is as follows:

$$
\begin{aligned}
& E\left(T_{i}\right)=\frac{1}{\mu_{i}-\lambda_{i}}, i=1,2,3 \\
& E(T)_{\text {all }}=\sum_{i=1}^{3} q_{i} E\left(T_{i}\right)
\end{aligned}
$$

where $q_{i}$ is the utilization rate of each tollbooth, $\lambda_{i}$ is the number of vehicles arriving in unit time in each tollbooth, $\mu_{i}$ is the number of vehicles served in unit time, and $E\left(T_{i}\right)$ is the average waiting time for a vehicle wait in a barrier toll. 
By searching correlative statistics information [13], the average traffic volume of the toll plaza per day $n$ is $80,000 \mathrm{pcu} / \mathrm{d}$. And the use proportion and traffic capacity of the three types tollbooths are listed in Table 1 and Table 2 respectively.

Table 1 The percentage of the three tollbooths users

\begin{tabular}{|c|c|c|}
\hline ETC tollbooths $q_{1}$ & Automated tollbooths $q_{2}$ & $\begin{array}{l}\text { Conventional tollbooths } \\
\qquad q_{3}\end{array}$ \\
\hline $40 \%$ & $30 \%$ & $30 \%$ \\
\hline
\end{tabular}

Table 2 The traffic capacity of the tree tollbooths

\begin{tabular}{ccc}
\hline ETC Tollbooths $\mu_{1}$ & Automated Tollbooths $\mu_{2}$ & $\begin{array}{c}\text { Conventional Tollbooths } \\
\mu_{3}\end{array}$ \\
\hline $1200 \mathrm{pcu} / \mathrm{h}$ & $360 \mathrm{pcu} / \mathrm{h}$ & $240 \mathrm{pcu} / \mathrm{h}$ \\
\hline
\end{tabular}

We can also get the parameters of the barrier toll in Table 3.

Table 3 Parameters estimates for different tollbooths

\begin{tabular}{cccc}
\hline $\begin{array}{c}\text { Three kinds of } \\
\text { tollbooths }\end{array}$ & ETC tollbooths & $\begin{array}{c}\text { Automated } \\
\text { tollbooths }\end{array}$ & $\begin{array}{c}\text { Conventional } \\
\text { tollbooths }\end{array}$ \\
\hline$\lambda$ & $n q_{1} / x_{1}$ & $n q_{2} / x_{2}$ & $n q_{3} / x_{3}$ \\
$\mu$ & $\mu_{1}$ & $\mu_{2}$ & $\mu_{3}$ \\
\hline
\end{tabular}

Suppose that the general construction of the barrier toll is unchanged, here we only introduce the different part.

In the improved cost model, the width of ETC tollbooths is different from the other two tollbooths. Hence, the added symbols are as follows.

Let $x_{1}$ be the number of ETC tollbooths, $x_{2}$ be the number of automated tollbooths, $x_{3}$ be the number of conventional tollbooths and $L_{E}$ be the width of ETC tollbooths.

The following formulas are used to analyze and establish the cost model.

(1) The width of the barrier toll:

$L_{N}=x_{1} L_{E}+\left(x_{2}+x_{3}\right)\left(L_{A}+L_{B}\right)$.

(2) The horizontal distance of the transition part BC:

$L_{B C}=\frac{1}{\beta}\left(L_{N}-M L_{M}\right)$.

(3) The total area of the toll plaza:

$S=L_{N}\left(L_{A B}+2 L_{B C}\right)-2 \times 0.5 \times L_{B C}\left(L_{N}-M L_{M}\right)$.

(4) The total cost:

Based on former discussion, we have

$p \approx \alpha S$.

Then the total cost can be expressed by

$p=\alpha\left\{L_{N}\left(L_{A B}+2 L_{B C}\right)-2 \times 0.5 \times \beta L_{B C}{ }^{2}\right\}+p_{0}$.

\section{Multi-objective Optimal Model}

We have already established the single-channel queuing model and the cost model, and obtained the average waiting time in the barrier toll as well as the total construction cost. In order to 
maximize the efficiency and benefit, the following multi-objective optimal model $M$ is established as follows.

The objective functions are $\min E(T)_{\text {all }}$

and

$\min p$

and the following constraint conditions must be satisfied

$0 \leq x_{1}, x_{2}, x_{3} \leq 10, x_{1}, x_{2}, x_{3} \in Z$.

\section{Solutions of Multi-objective Optimal Model Based on NSGA-II}

NSGA-II is to solve the multi-objective optimal model by using the genetic algorithm. Since genetic algorithm is one of global optimization approaches, which can be figured out multiple Pareto-optimal solutions. Thus it is an effective algorithm for solving the multi-objective models. In this paper, we apply NSGA-II to determine the solutions of founded multi-objective optimal model. The flow chat of algorithm is as follows.

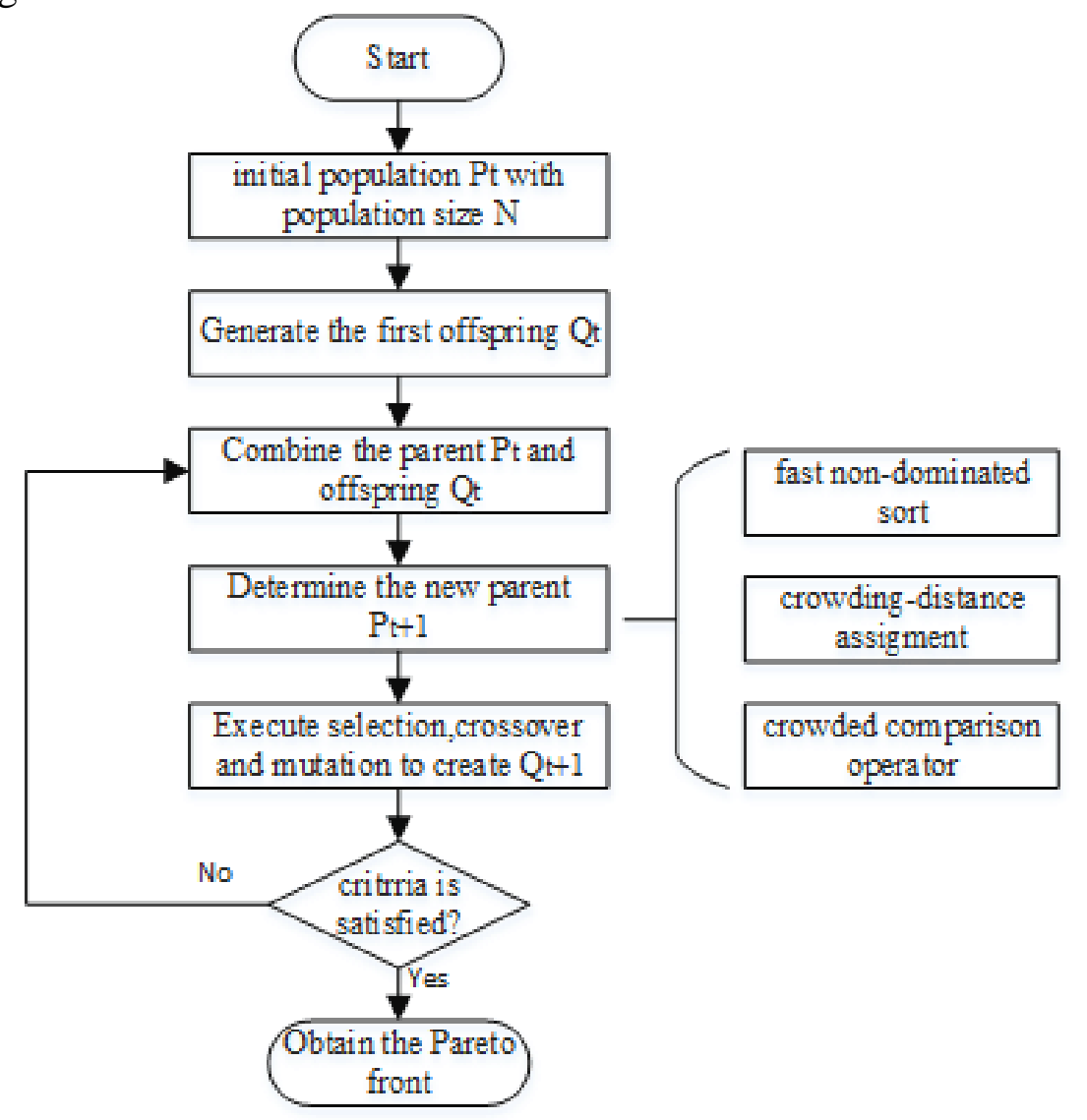

Figure 1. The flow chat of NSGA-II

The primary goal in implementing the genetic algorithm is to determine the genetic operator. There are 3 dependent variables in the objective function. Thus, the length of genetic operator is 3 . Meanwhile, the values of dependent variables are all restricted to the range $[1,10]$.

Here we use the tournament selection method to implement the genetic algorithm. Concretely, it excludes a certain percentage of inferior solutions in each small group, and remains non-inferior solutions and constitute a new population after crowding distance calculation and non-dominated sorting for combined population. Then we use the steps of crossover, mutation, select and fusion, which are repeated until satisfying the recursion terminal condition. Finally, an optimal set is obtained.

Now we set the parameters in the Table 4. 
Table 4 Parameters in genetic algorithm

\begin{tabular}{cccc}
\hline $\begin{array}{c}\text { Maximum } \\
\text { generation }\end{array}$ & $\begin{array}{c}\text { The optimal front-end } \\
\text { individual factor }\end{array}$ & $\begin{array}{c}\text { Population } \\
\text { size }\end{array}$ & $\begin{array}{c}\text { Fitness function } \\
\text { value deviation }\end{array}$ \\
\hline 200 & 0.3 & 200 & $1 \mathrm{e}-100$ \\
\hline
\end{tabular}

\section{Conclusions}

This paper presents an improved model base on $\mathrm{M} / \mathrm{M} / 1$ queue theory for designing the toll plaza by considering different ways of charging in the actual toll service system (human-staffed, automated and electronic toll collection), and applies the Non-dominated Sorting Genetic Algorithm II (NSGA-II) to optimize the design of lane number and charging mode layout. Our future work is to consider synthetically the prevention of accident, the traffic flow from the tollbooths to the lanes of travel and the total constructing cost of a toll plaza.

\section{Acknowledgments}

This work is supported by the Natural Science Foundation of Hubei Province (No. 2015CFB420).

\section{References}

[1] H.T. Abdelwahab, Traffic Micro-simulation Model for Design and Operational Analysis of Barrier Toll Stations, Ain Shams Engineering Journal (2016).

[2] U. Mahalingam, P.L. Manju, a Crowdsourcing Framework for Toll Plazas: Optimizing Delay at Toll Plazas (2016).

[3] C.L. Cheng, Study of capacity for ETC and MTC Hybrid Toll Station, Southwest Jiaotong University (2015).

[4] S.J. Zhao, The Charging Capacity and Key Designing Parameters of Freeway, Hebei University of Technology (2012).

[5] W.W. Liu, B. Lei, W. Ma, Z.B. Huang, Queuing theory model analysis of expressway toll station design, Communications Science and Technology Heilongjiang 4 (2013) 184-185.

[6] G. Liao, Analysis of expressway tollgate capacity: highway engineering, Highway Engineering 3 (2010) 153-172.

[7] J Li, L.L. Liu, B. Hu, J.C. Weng, R. Jian, Method of determining traffic capacity of mixed toll station based on simulation, Highway 7 (2012) 183-188.

[8] I. Corwin, S. Ganatra, N. Rozenblyum, A Single-Car Interaction Model of Traffic for a Highway Toll Plaza: UMAP Modul (2016).

[9] J.D. Qin, X.W. Liu, Witold Pedryczc, An extended TODIM multi-criteria group decision making method for green supplier selection in interval type-2 fuzzy environment, European Journal of Operational Research 258 (2017) 626-638.

[10] J.J.H. Liou, Y.C. Chuang, G.H. Tzeng, A fuzzy integral-based model for supplier evaluation and improvement, Information Sciences 266 (2014) 199-217. 